Review Articles

\title{
Gene Transfer Vector Derived from Jembrana Disease Virus: A Review
}

\author{
${ }^{1,2}$ Asmarani Kusumawati, ${ }^{3}$ Tenri A. Wanahari, ${ }^{4}$ Pudji Astuti, ${ }^{3}$ Basofi A. Mappakaya and ${ }^{5}$ Wasito \\ ${ }^{1}$ Departement of Reproduction, Faculty of Veterinary Medicine, \\ Gadjah Mada University, Jalan Fauna No.2, Yogyakarta, 55281 Indonesia \\ ${ }^{2}$ Centre of Biotechnology Study, Gadjah Mada University, Jalan Teknika Utara, Yogyakarta, 55281 Indonesia \\ ${ }^{3}$ Faculty of Medicine, Sebelas Maret University, Jalan Ir. Sutami 36 A, Surakarta, 57126 Indonesia \\ ${ }^{4}$ Departement of Physiology, Faculty of Veterinary Medicine, \\ Gadjah Mada University, Jalan Fauna No.2, Yogyakarta, 55281 Indonesia \\ ${ }^{5}$ Departement of Pathology, Faculty of Veterinary Medicine, \\ Gadjah Mada University, Jalan Fauna No.2, Yogyakarta, 55281 Indonesia
}

Article history

Received: 20-05-2015

Revised: 07-08-2015

Accepted: 10-08-2015

Corresponding Author: Asmarani Kusumawati Departement of Reproduction, Faculty of Veterinary Medicine, Gadjah Mada University, Jalan Fauna No.2, Yogyakarta, 55281 Indonesia

Tel: +6281328867709;

+6274560863 ;

Fax: +6274560863

Email: kartapati_2008@yahoo.com

\begin{abstract}
The etiological agent of Jembrana disease is a virus which was accordingly named Jembrana Disease Virus (JDV). JDV was a member of the family of Retroviridae, of the subfamily of lentiviruses. As a member of the lentivirus, JDV has general property to integrate their genome into the chromosomal DNA of the host-cells. It is one of their common properties which is not shared by simple retroviruses such as murine leukimia virus or avian sarcoma virus. Lentiviruses are highly speciesspecific and share the property of replicating in dividing and non-dividing terminally differentiated cells. JDV-based gene transfer vector which had been developed is appeared to possess a broad tropism and a unique capacity of mediating efficient gene transfer. This review summarize JDV-based viral vector system that has been developed. The main principles and components of JDV viral gene transfer system and its implication in the future research will be discussed.
\end{abstract}

Keywords: Jembrana Disease Virus, Lentiviral Vector, Gene Delivery System, Gene Transfer System

\section{Introduction}

Jembrana disease poses the major problem in Bali cattle industry especially in Indonesia and Australia due to the high mortality of the infected cattle, resulting in economic loss (Chadwick et al., 1998; Soesanto et al., 1990). The etiological agent, Jembrana Disease Virus (JDV), belongs to the family of Retroviridae, of the subfamily of lentiviruses (Kusumawati et al., 2014a). Lentiviruses have the ability to integrate their genome into the chromosomal DNA of the host-cells. It is one of their common properties which is not shared by simple retroviruses such as murine leukimia virus or avian sarcoma virus (Naldini and Verma, 2000). Lentiviruses are highly species-specific and share the property of replicating in dividing and non-dividing terminally differentiated cells (Olsen, 2001). They are able to do this because they have two virion proteins (matrix and $\mathrm{Vpr}$ ) that interact with the nuclear import machinery and can transport the pre-integration complex across the nuclear membrane from the cytoplasm to the nucleus in the absence of mitosis (Naldini et al., 1996). In addition, monocyte-macrophage lineage cells are targets of all lentiviruses. Hence, lentiviral infection implicated in immune system accounts for dissemination throughout the body and characterized by multi-organ involvement as the virus may cause CD4 lymphocytes depletion and immunodeficiency. Nevertheless following the virus type considered, the progression of infection may be rapid, i.e., within weeks as with Equine Infectious Anaemia Virus (EIAV) (Olsen, 1998; 2001) or Jembrana Disease Virus (JDV) (Wilcox et al., 1995) or takes months to years to develop as with maedi/visna virus, Caprine Arthritis Encephalitis Virus (CAEV) or Human Immunodeficiency Virus (HIV) (Olsen, 2001). Lentiviruses that infect non-primates share some features with HIV and other primate viruses. The distinguishing features are found in the genome organization and viral gene function. As already 
indicated to typical lentiviruses, the genome comprises Long Terminal Repeats (LTRs) at both ends; major structural genes gag, pol, env, encoding structural and enzymatic proteins that make up infectious viral particles; and at least the accessory genes vif, tat, rev, encoding proteins that regulate gene expression, as shown in Fig. 1 (Chadwick et al., 1995; Kusumawati et al., 2014a; Westendorp et al., 1995). Gene transfer systems have been developed for 3 primate lentiviruses and 5 non-primate lentiviruses (Metharom et al., 2000; 2001; Olsen, 1998; 2001; Wang et al., 1994). This review deals with gene transfer system derived from JDV which has been developed (Metharom et al., 2000; 2001). The main principles and components of JDV viral gene transfer system and its implication in the future research will be discussed.

\section{Principle of Lentiviral Gene Transfer System}

Lentiviruses are enveloped single-stranded RNA viruses, which have been widely used in gene delivery protocol. Lentiviruses have a genome of about 7-10 kb, composed several structural genes regions termed gag (encode for structural capsid protein), pro (encode for viral protease), pol (encode for integrase and viral reverse transcriptase and env (encode for envelope glycoprotein). The genome also has the accessory genes vif, tat, rev, encoding proteins that regulate gene expression and cis-acting sequence, termed Long Terminal Repeats (LTR) at both ends, which have a role in transcriptional control and integration (Fig. 1).

The basic concept of lentiviral gene transfer system is the production of disabled, replication defective transfer vectors that allow the expression of genes of interest. This gene transfer vector system design is based on separation of cis-acting elements in the genome from sequences encoding trans-acting proteins (Metharom et al., 2000; 2001; Olsen, 2001). The system has three elements: (i) a bicistronic transfer vector plasmid, (ii) a packaging plasmid or protein expression cassette plasmid and (iii) an envelope plasmid. Separately, none of these three plasmids is able to produce transfer vectors yet the bicistronic transfer vector will only be packaged into the final RNA viral particles as it contains viral cis-acting elements required for replication and integration. Afterward, the two remaining plasmids are required to assemble, package and encapsidate. It is due to the expression of viral protein cassette that provides the genes encoding the viral proteins necessary for assembling the RNA form of the gene transfer vector into viral particles whereas the envelope expression plasmid supplies the envelope for encapsidation. The gene transfer vector plasmid may also includes "foreign" genes, i.e., genes of interest, to be expressed in target cells for defined purposes.

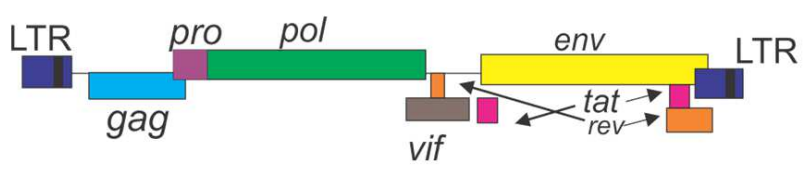

Fig. 1. Schematic structure of Jembrana disease virus genome. It contains the structural genes gag, pro, pol and env which encode structural and enzymatic proteins that make up infectious viral particles; flanking Long Terminal Repeats (LTR) that characterize all retroviruses; and a number of accessory genes, including tat, rev and vif which are required for the production of regulatory proteins

The system works separately by the expression of the three plasmids that produce various viral components in packaging cells, followed by transient co-transfection in suitable cells to finally make up the whole transfer system, the final RNA viral particles which unable to replicate and can express exogeneous genes.

\section{Design of the Components of JDV Transfer System}

The JDV-based gene transfer vector is produced from three initial components. Their characteristics are described below (Metharom et al., 2000; 2001).

\section{Bicistronic Transfer Vector Plasmid}

The bicistronic transfer component contains cissequences, including 5'- and 3'-LTRs, non functional gag and env gene, internal CMV (cytomegalovirus) promoter driving a GFP-IRES-neo cassette and two Multicloning Sites (MCS). The contents of the bicistronic transfer component have their own contribution in making the product of the system. Firstly, the introduced Internal Ribosome Entry Site (IRES) which provides a second entry site for ribosomes and allows the simultaneous translation of a second gene, i.e., the gene for neomycin resistance, in addition to the gene coding for Enhanced Green Fluorescence Protein (EGFP), used as reporter, in testing experiments. This function is also supported by the neo cassette that provides an antibiotic selection while the MCS are intended for the insertion of exogenous gene/s and expression of protein/s of interest. Conversely, the plasmid is disable to generate replication-competent viral particles because the env, vif, tat, rev genes were removed while gag and $e n v$ genes were truncated. Due to the non functional env gene, the envelope proteins will not be synthesized from this plasmid. A partial sequence of gag was retained in the construct to ensure efficient encapsidation and/or RNA stability (Mitrophanous et al., 1999; Olsen, 1998) though the start codon was mutated. The bicistronic transfer vector is the only that will be encapsidated into the final transfer system product (Fig. 2). 


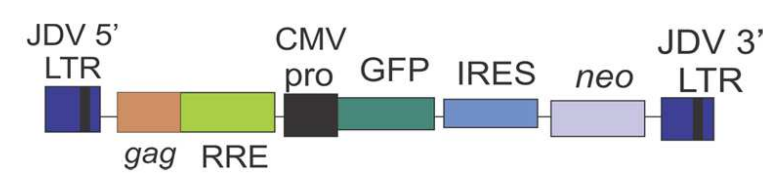

Fig. 2. Schematic structure of Jembrana disease virus transfer vector system. The component contains: 5'- and 3'-LTRs (long terminal repeats), non functional gag genes, RRE (rev responsive element), internal CMV (cytomegalovirus) promoter and GFP-IRES-neo cassette (GFP: Green fluorescence protein; IRES: Internal ribosome entry site; neo: Gene for neomycin resistance)

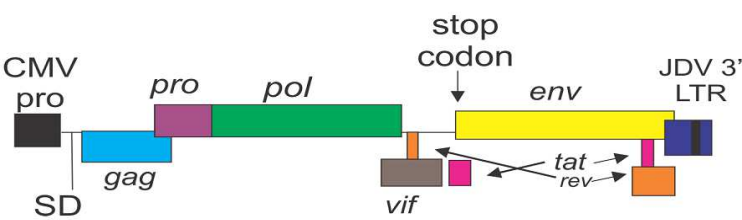

Fig. 3. Schematic structure of Jembrana disease virus protein expression packaging system. The packaging system with one Splice Donor (SD) which related to internal CMV promoter contains all of the JDV viral genes including trans sequences gag, pro, pol, vif, tat, rev genes, non-functional env gene and 3'-LTR

\section{Packaging Plasmid or Protein Expression Cassette Plasmid}

The protein expression cassette is used for the expression of viral proteins. It should be devoid of all cis-acting elements including the RNA encapsidation signal and cis-acting elements implicated in DNA synthesis and integration. Along with several modifications that have been made to disable the construct replication, the packaging plasmid contains all of the viral genes including trans sequences gag, pol, vif, tat, rev genes but devoid of functional env gene to prevent the production of JDV envelope. Deletion of certain sequences of gag gene is intended to disable its packaging activity. JDV LTR is replaced by CMV promoter to drive the expression of viral proteins required in trans-sequences. Overall, the packaging plasmid alone does not provide, in se, elements required to generate replication competent viral particles (Fig. 3).

\section{Envelope Plasmid}

As both the transfer and the packaging plasmids cannot provide the necessary elements for packaging, an envelope-supplying plasmid constitutes the third component. It contains the gene coding for $\mathrm{G}$ protein of Vesicular Stomatitis Virus (VSV), i.e., VSV-G, to supply the vector an envelope for pseudotyping and extending the host range to human cells (Olsen, 1998). The substitution of JDV envelope with VSV-G envelope also conferred particle stability, enabling the concentration of viral particles by ultracentrifugation.

\section{Production of Disabled, Replication- Defective Transfer Vector}

The final purpose of the transfer system construction is to obtain replication-defective transfer vectors allowing the expression of genes of interest in target and which are integrated into the genomic DNA of the hostcells (Metharom et al., 2000; 2001). The final products resulting from the three initial components are VSV-G pseudotyped JDV lentivirus vectors, i.e., JDV RNA viral particles with VSV-G envelope that are disabled in replication for they are devoid of gag, pol, env, vif, tat and rev. They are produced by transient co-transfection of the 3 plasmids in the human embryonic 293T cells. Finally, several parameters were examined to determine whether the produced JDV-based RNA viral vectors can actually function as gene transfer vector.

\section{Gene Expression from JDV-Based Viral Vector}

\section{In Vitro Transduction of Target Cells}

Transduction and expression of the reporter gene EGFP was examined by transducing various cell types with JDV-based viral vectors obtained by transient cotranfection of the 3 plasmids. Transduction, as judged by G418 resistance and EGFP fluorescence, was possible in several types of cell line, i.e., human embryonic kidney cells 293 and 293T, human cancer HeLa cells, monkey COS7, murine melanoma cell line B16, primary Fetal Bovine Lung (FBL) cells, with an efficacy of $28-78 \%$ following the cell type. This transduction process has the highest efficiency at the observation in FBL. Overall, the JDV-based vectors are able to mediate gene transfer to cells originating from bovine, primate, murine and human sources, including primary and transformed cells and interestingly both replicating and growth-arrested as attested by the successful transduction in aphidicolin-treated HeLa cells (Metharom et al., 2000; 2001).

\section{Transgene Expression and Integration}

Following transduction of cells with JDV viral vectors, the observation of EGFP fluorescence, i.e., the reporter protein in testing experiments, showed that EGFP is only expressed $24 \mathrm{~h}$ post transduction and its expression intensifies gradually in positively transduced cells. This result is agreeing with transgene expression, but not with EGFP protein transfer. The result is also in favour of transgene integration in the genomic DNA which will result in stable expression of the marker gene EGFP. Indeed, in repeatedly passaged positive clones, the level of EGFP expression stays high throughout every passage, with no decay or change in intensity in nucleus and cytoplasm. 
Transgene integration is finally corroborated by PCR analysis of genomic DNA using specific primers that allow to distinguish the emergence of replication competent JDV from transgene integration. Primers specific for parts of exon-1 tat, present in the packaging plasmid, but absent in both the transfer plasmid and the envelope plasmid, will reveal the emergence of replication JDV whereas transgene integration will be revealed by primers detecting the $\mathrm{U} 3$ and $\mathrm{R}$ region of 3'-LTR contained in the bicistronic transfer plasmid. The presence of $\mathrm{U} 3$ and $\mathrm{R}$ region in the packaged VSVG pseudotyped JDV vector was revealed on PCR analysis which also in contrary proved that the vector does not contain the tat gene. The appearance of U3 and $\mathrm{R}$ region made the bicistronic transfer vector is actually integrated in the genomic DNA somehow. This integration which is mediated by $\mathrm{U} 3$ and along with LTR contributed to stable gene transfer. Otherwise, lack integrating elements such as the packaging plasmid and the envelope plasmid should not be integrated in the genomic DNA. The testing experiments also showed that the JDV transfer vector is able to be efficiently packaged and encapsidated with VSV-G envelope with JDV native LTR. Furthermore, due to the highly efficient transcription activity of JDV LTR during the packaging process, high titres of vectors are produced in the packaging $293 \mathrm{~T}$ cells (Metharom et al., 2000; 2001).

In order to avoid any undesirable side effect, the packaged JDV vectors should also be disabled for replication and exhibiting reduced chances of recombination. This is attained by devoid the viral gene for gag, pol, env, vif, tat and rev in the final packaged JDV vectors. The replication disability of the vector was further attested by transduction of these vectors in JDV permissive FBL cells or in JDV non permissive 293 T cells. It worked out that both cell types did not generate replication-competent vectors. Indeed, although they are EGFP-positive themselves, when the supernatant of these cells are used to transduce naive FBL or $293 \mathrm{~T}$ cells, no EGFP-positive cells were obtained $24 \mathrm{~h}$ posttransduction and after three passages. It is crystal clear that the JDV vectors produced by co-transfection of the initial three plasmids are not replication-competent due to the EGFP monitoring that also gives negative response for vectors harvested from JDV permissive FBL cells (Metharom et al., 2000; 2001).

Among the three initial components, the bicistronic transfer vector is the only that is encapsidated into the final transfer system product. The resulting gene transfer vector is lack of genes necessary for replication. However, the presence of certain essential cis-acting sequences is maintained to enable the reverse transcription and the integration into the host cell chromosome. These sequences include the a packaging signal sequence $(\psi)$, the Primer Binding Site (PBS), the terminal repeat $(\mathrm{R})$ and the purine-rich region $5^{\prime}$ of the 3'-LTR. These elements are essential for the reverse transcription process especially in HIV-1 (Gasmi et al., 1999). The $\psi$ sequence will ensure the encapsidation of RNA vector into the viral particle vector. The efficiency of encapsidation will also be amplified by the 3'-env region as in HIV-1-based vectors (Naldini et al., 1996). As in HIV-1 DNA plasmid, the putative RRE (rev (regulator of virus expression) responsive element) was also added to JDV vectors to facilitate the expression of conserved viral proteins (Fig. 2) (Qiu et al., 1999; Schneider et al., 1997). Consequently, the JDV vector may potentially lead to the development of bovine vaccines for JDV that are able to elicit both humoral and cellular immune responses as efficiently as livevirus vaccines but without the pathogenic consequences of live viruses because the vectors is defective (Metharom et al., 2000; 2001).

\section{Conclusion}

An integrated, disabled, replication-defective transfer vector, based on JDV, i.e., JDV RNA viral particles with VSV-G envelope, can be formulated by co-transfection of its initial three components, i.e., the bicistronic transfer vector plasmid, the packaging plasmid and the envelope plasmid, in the human embryonic $293 \mathrm{~T}$ cells (Metharom et al., 2000; 2001). Once packaged, only the bicistronic transfer plasmid is present in the final formed vector and in JDV vector transduced cells. This final formed vector does not contain the packaging plasmid and the third component, the envelope plasmid, merely takes part of supplying the VSV-G envelope. The transfer system appears to possess a broad tropism and a unique capacity of mediating efficient gene transfer and stable expression of the marker genes over a long period of time, due to the transgene integration in the genomic DNA.

The design and construction of the transfer vectors should facilitate the study of lentivirology and pathogenesis associated with JDV or other bovine virus infections (Kusumawati et al., 2014b). The vector can be used for example to unravel and reveal the mechanisms of interaction of JDV with its host-cells. The disabled, replication defective JDV-based vectors suit the inclusion and expression of genes corresponding to JDV specific proteins such as gag or env whose expression in a DNA plasmid is problematic (Schneider et al., 1997; Schwartz et al., 1992). They have so the potential contribution for the development of viral bovine vaccines for JDV that can elicit both humoral and cellular immune responses. This approach is also relevant to the study of other bovine diseases. 


\section{Acknowledgement}

We acknowledged to Rizqa Febriliany Putri for provided the general support and aid.

\section{Funding Information}

This work was partly funded by a grant from Directorate General of Higher Education (DIKTI), Ministry of Education and Culture of Indonesia.

\section{Author's Contribution}

Asmarani Kusumawati: Designed the literature review study, participated in aquisition of data, coordinated and contributed to the writing of the manuscript.

Tenri Ashari Wanahari: Participated in aquisition of data, contributed to the writing of the manuscript and designed the graphical arts.

Pudji Astuti: Participated in aquisition of data and provided technical guidance of the literature review study.

Basofi Ashari Mappakaya: Contributed to the writing of the manuscript.

Wasito: Provided technical guidance of the literature review study.

\section{Ethics}

This study was approved by Research Ethics Commitee, Faculty of Veterinary Medicine, Gadjah Mada University, Indonesia.

\section{References}

Chadwick, B.J., R.J. Coelen, G.E. Wilcox, L.M. Sammels and G. Kertayadnya, 1995. Nucleotide sequence analysis of Jembrana disease virus: A bovine lentivirus associated with an acute disease syndrome. J. Gen. Virol., 76: 1637-1650.

PMID: 9049370

Chadwick, B.J., M. Desport, J. Brownlie, G.E. Wilcox and D.M. Dharma, 1998. Detection of Jembrana disease virus in spleen, lymph nodes, bone marrow and other tissues by in situ hybridization of paraffinembedded sections. J. Gen. Virol., 79: 101-106. PMID: 9460930

Gasmi, M., J. Glynn, M.J. Lin, D.J. Jolly and J.K. Yee et al., 1999. Requirements for efficient production and transduction of human immunodeficiency virus type 1-based vectors. J. Virol., 73: 1828-1834.

PMID: 9971760

Kusumawati, A., T.A. Wanahari, R.F. Putri, B.A. Mappakaya and I.D. Tampubolon, 2014a. The structure and function of jembrana disease virus genome. J. Inf. Mol. Biol. Sci., 2: 26-29.
DOI: 10.14737/jimb.2307-5465/2.2.26.29

Kusumawati, A., T.A. Wanahari, R.F. Putri, T. Untari and T. Hartati et al., 2014b. Clinical and pathological perspectives of Jembrana disease virus infection: A review. Biosci. Biotech. Res. Asia., 11: 1221-1225. DOI: $10.13005 / \mathrm{bbra} / 1509$

Metharom, P., S. Takyar, H.H. Xia, K.A. Ellem and J. Macmillan et al., 2000. Novel bovine lentiviral vectors based on Jembrana disease virus. J. Gene Med., 2: 176-185. PMID: 10894263

Metharom, P., S. Takyar, H.Q. Xia, K.A. Ellem, G.E. Wilcox and M.Q. Wei, 2001. Development of disabled, replication-defective gene transfer vectors from the Jembrana disease virus, a new infectious agent of cattle. Vet. Microbiol., 3: 9-22.

PMID: 11278119

Mitrophanous, K., S. Yoon, J. Rohll, D. Patil and F. Wilkes et al., 1999. Stable gene transfer to the nervous system using a non-primate lentiviral vector. Gene Ther., 6: 1808-1818. PMID: 10602376

Naldini, L., U. Balmer, P. Gallay, D. Ory and R. Mulligan et al., 1996. In vivo gene delivery and stable transduction of nondividing cells by a lentiviral vector. Science, 272: 263-267. PMID: 8602510

Naldini, M. and I.M. Verma, 2000. Lentiviral vectors. Adv. Virus Res., 55: 599-609. PMID: 11050959

Olsen, J.C., 1998. Gene transfer vectors derived from equine infectious anemia virus. Gene Ther., 5: 1481-1487. PMID: 9930301

Olsen, J.C., 2001. EIAV, CAEV and other lentivirus vector systems. Somat. Cell Mol. Genet., 26: 131-45. PMID: 12465465

Qiu, J.T., R. Song, M. Dettenhofer, C. Tian and T. August et al., 1999. Evaluation of novel human immunodeficiency virus type 1 Gag DNA vaccine for protein expression in mammalian cells and induction of immune response. J. Virol., 73: 9145-9152. PMID: 10516021

Schneider, R., M. Campbell, G. Nasioulas, B.K. Felber and G.N. Pavlakis, 1997. Inactivation of human immunodeficiency virus type 1 inhibitory elements allow Rev-independent expression of Gag and Gag/protease and particle formation. J. Virol., 71: 4892-4903. PMID: 9188551

Schwartz, S., M. Campbell, G. Nasioulas, J. Harrison and B.K. Felber et al., 1992. Mutational inactivation of an inhibitory sequence in human immunodeficiency virus type 1 results in Rev-independent gag expression. J. Virol., 66: 7176-7182. PMID: 1433510

Soesanto, M., S. Soeharsono, A. Budiantono, K. Sulistyana and M. Tenaya et al., 1990. Studies on experimental Jembrana disease in Bali cattle. II. Clinical signs and haematological changes. J. Comp. Pathol., 103: 61-71. PMID: 2394847 
Wang, S.Z., K.E. Rushlow, C.J. Issel, R.F. Cook and S.J. Cook et al., 1994. Enhancement of EIAV replication and disease by immunization with a baculovirus-expressed recombinant envelope surface glycoprotein. Virology, 199: 247-251. PMID: 8116252

Westendorp, M.O., R. Frank, C. Ochsenbauer, K. Striker and J. Dhein et al., 1995. Sensitization of T cells to CD95-mediated apoptosis by HIV-1 Tat and gp120. Nature, 375: 497-590. PMID: 7539892
Wilcox, G.E., B.J. Chadwick and G. Kertayadnya, 1995. Recent advances in the understanding of Jembrana disease. Vet. Microbiol., 46: 249-255.

PMID: 8545963 